\title{
New Molecular Tools for Protein Detection and Dynamics in Electron Microscopy
}

Daniela Boassa ${ }^{1,2}$, John T. Ngo ${ }^{3}$, Mason R. Mackey ${ }^{1,2}$, Ranjan Ramachandra ${ }^{1,2}$, Thomas J. Deerinck ${ }^{1,2}$, Mark H. Ellisman ${ }^{1,2}$ and Stephen R. Adams ${ }^{4 *}$

${ }^{1}$. Department of Neurosciences, University of California San Diego, La Jolla, CA, USA.

2. National Center for Microscopy and Imaging Research, University of California San Diego, La Jolla, CA, USA.

3. Department of Biomedical Engineering and Biological Design Center, Boston University, Boston, MA USA.

4. Department of Pharmacology, University of California San Diego, La Jolla, CA, USA.

* Corresponding author: sadams@ucsd.edu

Genetically-encoded tags such as miniSOG and APEX have become valuable tools for marking protein localization by deposition of osmiophilic DAB for electron microscopy (EM) [1,2]. To visualize proteinprotein interactions, we have now developed split miniSOG, analogous to the well-known fluorescent complementation assay with a split fluorescent protein but generating an EM-visible mark by photooxidation of DAB. We identified two fragments, N-terminal segment termed "mSOG1-94" $(10.96 \mathrm{kDa})$ and a 46 amino acid C-terminal polypeptide designated "mSOG-Ja95-140" (5.15 kDa) that when fused to interacting proteins enable binding of FMN, the endogenous fluorescent photosensitizer. We have demonstrated its reconstitution with leucine zipper domains of bFos and bJun, subunits of the AP-1 transcriptional complex that interact constitutively as nuclear heterodimers, and visualized neurotoxic assemblies of $\alpha$-synuclein, a neuronal protein involved in Parkinson's disease.

We recently introduced a method for highlighting multiple specific proteins and distinguishing them by electron energy-loss spectroscopy (EELS) to give a version of color EM [3]. Briefly, proteins are labeled with antibodies, genetically-encoded tags (miniSOG or APEX) and localized precipitates of lanthanide (Ln) chelates of DAB are sequentially formed by orthogonal activation of the photosensitizers or peroxidases. Energy-filtered transmission EM (EFTEM) gives lanthanide elemental maps that can be overlaid on a conventional TEM to reveal the desired proteins localization. To increase the methods sensitivity, we have developed $2^{\text {nd }}$ generation DAB-chelates, that precipitate $>4$ times more lanthanide than their predecessors, with histone $\mathrm{H} 2 \mathrm{~B}$ nuclear and with mitochondrial matrix using Ce2-DAB. Additionally, we have devised a new strategy that involves precipitation of a DAB-alkyne followed by a reaction to a lanthanide-chelate azide. This bypasses the difficulty in precipitating polar DAB Ln chelates and expands our choice of known metal chelates, including potentially short polymers of them that would prevent $\mathrm{DAB}$ precipitation if attached before (photo)oxidation. These methods give greater metal deposition than our first-generation Ln-DAB2 enabling the collection of multi-tilt EELS tomograms and multi-spectral EELS imaging of the more intense 'low loss' spectral peaks that permit shorter acquisitions (less beam damage), greater signal (e.g. for tomograms) and potentially new multi-color modes for SEM.

The use of peroxidases in LM and EM to generate localized deposition includes exogenous horse radish peroxidase (HRP) and more recently genetically-encoded ascorbate peroxidases, APEX. We have now adapted a small molecule peroxidase based on a ferric-macrocycle (Fe-TAML) to EM that is very small (M.W. 500); about 100 times smaller than HRP and the size of a fluorophore molecule, but capable of generating an EM visible reaction product, and this is an advantage in terms of penetration into fixed cells and tissues for 3D labeling. To demonstrate its use, we used a synthesized azide derivative that can be 
used in "Click-EM" for imaging nucleic acids, lipids and glycans by cellular incorporation of metabolic analogs functionalized with azides or alkynes [4]. Photoreactive fluorescent dyes and now a smallmolecule peroxidase can be specifically ligated to these analogs with $\mathrm{Cu}(\mathrm{I})$-catalyzed azide-alkyne cycloaddition (CuAAC), a reaction often referred to as "click chemistry." Localized generation and precipitation of oxidized, osmiophilic DAB labels the structures and enables their visualization by EM. We metabolically labeled DNA by incorporation of 5-ethynyl-2-deoxyuridine in cultured cells overnight followed by click-chemistry attachment of FeTAML-azide. After incubation with DAB and $\mathrm{H}_{2} \mathrm{O}_{2}$ and a robust reaction product was observed by light microscopy and after processing for EM, high-resolution electron micrographs (Figure 1) showed DNA labeling patterns similar to what we obtain using other methods for marking DNA [5]. We have also applied this peroxidase to immunolabeling by conjugation to secondary antibodies and demonstrated tubulin labeling in cultured cells. Genetically-targeting this artificial peroxidase is also possible as demonstrated with HaloTag for visualizing histone H2B [6].

\section{References:}

1. X Shu et al., PLOS Biology 9, e1001041

2. SS Lam et al., Nature Methods 12, p. 51

3. SR Adams et al., Cell Chem. Biol. 23, p. 1417

4. JT Ngo et al., Nature Chem. Biol. 12, p. 459

5. HD Ou et al., Science, 357, eaag0025

6. Supported by NIH grants GM86197, GM103412
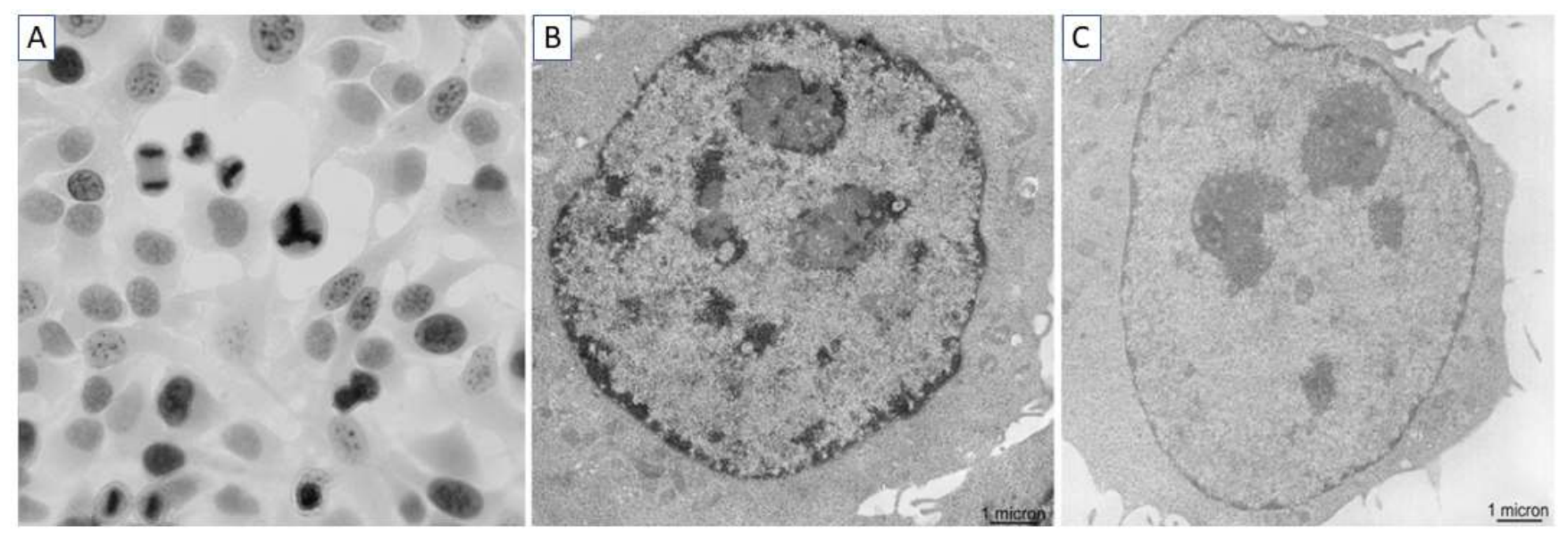

Figure 1. Light (A) and EM (B) labeling of DNA in HeLa cells using metabolic labeling of DNA with 5ethynyl-2-deoxyuridine for $12 \mathrm{~h}$ followed glutaraldehyde fixation and by click-chemistry attachment of Fe-TAML azide via CuAAC. Cells were incubated with DAB and $\mathrm{H}_{2} \mathrm{O}_{2}$ for $2 \mathrm{~min}$. Cells were stained with OsO4, embedded in resin, and thin sectioned for EM. C) Control cell without incorporation of 5-ethynyl2-deoxyuridine but otherwise identically treated. 